\title{
Clinician engagement is critical to public engagement with clinical trials
}

\author{
Christopher J Stock head of research and development communications and strategy ${ }^{1}$, Nick Carley \\ managing director ${ }^{2}$, Ben Hickman research director ${ }^{2}$, John Primrose professor of surgery ${ }^{3}$, Alison \\ Ayres director of communications and public engagement ${ }^{1}$, Christine McGrath director of research \\ and development ${ }^{1}$, Christopher J Edwards associate director ${ }^{4}$, Saul N Faust director ${ }^{4}$
}

\footnotetext{
${ }^{1}$ University Hospital Southampton NHS Foundation Trust, Southampton SO16 6YD, UK; ${ }^{2}$ Alterline Research, Manchester M4 6JW; ${ }^{3}$ University of Southampton Faculty of Medicine, Southampton SO16 6YD; ${ }^{4}$ Southampton NIHR Wellcome Trust Clinical Research Facility, Southampton SO16 6YD
}

Five years on from Godlee and Chalmers' advocacy of greater patient information to improve access to clinical trials, ${ }^{1}$ we have conducted research showing that engaging clinicians with local research and trial information is as critical to enabling participation as are public engagement and information provision.

Our study explored the perceptions, motivations, and barriers to trial participation among 1101 adult members of the public and among 26 consultants, primary care physicians, and nurses across southern England.

Some $90 \%$ of public respondents agreed that NHS supported research was important, and $47 \%$ were likely to take part in trials if asked, but only $10 \%$ had ever participated. Importantly, respondents said that they would be more likely to take part if their own NHS clinicians were explaining the research; however, research was discussed in only $5 \%$ of consultations in the previous 12 months, and only $15 \%$ of respondents could recall discussing trials with a clinician at any time.

Primary care physicians and hospital consultants perceive themselves and peers either as "researchers"- an academically orientated minority—or as "practitioners" focused on care provision. While the "practitioners" were positive about research and were happy to refer patients to trial, they lacked accessible and reliable local information needed to do so.

Time and workload pressures were key barriers to discussing research with patients, in terms of finding and assessing trial information and of building discussion into consultations, while the perceived bureaucratic burdens of research governance limited people's pursuit of research.

The finding that $95 \%$ of clinicians are not doing so, when they are inherently trusted to explain trial options by a clearly willing public, is critical if we are to achieve increased engagement with research. It is clear that further targeting of resources towards clinician engagement is needed, alongside management and education interventions to make communication about trials a routine part of all NHS consultations.

Competing interests: None declared.

Full response at: www.bmj.com/content/340/bmj.c725/rr.

1 Godlee F, Chalmers I. Publishing information about ongoing clinical trials for patients. BMJ 2010;340:c725.

Cite this as: $B M J$ 2015;350:h3140

๑ BMJ Publishing Group Ltd 2015 